\title{
Indicadores Antropométricos en un Centro de Acondicionamiento Físico Urbano de la Provincia de Heredia
}

\author{
Anthropometric Indicators in an Urban Fitness Center of the Province of Heredia \\ Gustavo Rivera Cabezas y Pedro Ureña Bonilla \\ Escuela Ciencias del Movimiento Humano y Calidad de Vida, Universidad \\ Nacional, Costa Rica. \\ gustavo.rivera.cabezas@una.cr
}

\begin{abstract}
Resumen
El propósito de este estudio fue identificar los valores antropométricos y su relación con la salud en los usuarios del Centro de Acondicionamiento Físico (CAF) de la Escuela de Ciencias de la Salud y Calidad de Vida (CIEMHCAVI) de la Universidad Nacional. Se analizó 1000 expedientes, aunque por razones de ausencia de datos se trabajó con 563. La información recopilada correspondió a 315 hombres y 248 mujeres con edad promedio de 28,5 $\pm 11,3$ y de 30,2 \pm 11,3 años, respectivamente. Cada expediente contenía información sobre los valores antropométricos índice de masa corporal (IMC), peso, porcentaje de grasa, circunferencia de cintura y cadera. Los resultados relativos al porcentaje de grasa corporal e IMC correspondieron a una población sana según parámetros internacionales. Tanto hombres como mujeres mostraron IMC similares, aunque las mujeres evidenciaron valores promedio significativamente superiores a los hombres en cuanto al porcentaje de grasa y riesgo cardiovascular. Asimismo, se registró correlaciones positivas y significativas entre IMC, porcentaje de grasa y relación cintura cadera. En general, los datos analizados evidenciaron valores antropométricos saludables para la mayoría de la población que asistió al CAF de la CIEMHCAVI en el periodo comprendido entre el 2004 - 2006.
\end{abstract}

Palabras Claves: Centro de acondicionamiento físico, Entrenamiento de contrarresistencia, Evaluación antropométrica, Indicadores antropométricos, Riesgo cardiovascular.

\begin{abstract}
The aim of this study was to identify trends in anthropometric values and their relation with health in users of the Centre for Physical Fitness (CPF) of the School of Health Sciences and Quality of Life (CIEMHCAVI) of the National University. 1000 records were analyzed, although for reasons of lack of data we only worked with 563. The information gathered corresponded to 315 men and 248 women with an average age of $28.5 \pm 11.3$ and $24830.3 \pm 11.3$ years respectively. Each file contained information on the following anthropometric values: Body Mass Index (BMI), weight, fat percentage, and waist to hip ratio. The results for percentage of body fat and BMI corresponded to a healthy population by international standards. Both men and women showed similar BMI, while women showed significantly higher mean values than men for the percentage of body fat and cardiovascular risk. Furthermore, significant positive correlations were recorded between BMI, body fat percentage and waist to hip ratio. Overall, the data analyzed showed healthy anthropometric values for most of the people attending the CPF - CIEMHCAVI in the period from 2004 to 2006.
\end{abstract}

Key Words: Center of Physical Fitness, Strength Training, Anthropometric Assessment, Anthropometric Indicators, Cardiovascular Risk. 


\section{Introducción}

El valor principal del registro de indicadores antropométricos estriba en que permite predecir el riesgo de enfermedades crónicas, identificar el estado nutricional de las personas y comparar los patrones de salud en distintas poblaciones a lo largo del tiempo (Arruda, Martins, Machado, Santos de Moraes, De Carvalho, 2010; World Health Organization, 2000). Entre los indicadores antropométricos más utilizados se reportan: el Índice de Masa Corporal (IMC), el porcentaje de grasa corporal y la relación del índice cintura cadera (Martínez, Carmenate, Díaz, Toledo, Prado y otros, 2009).

El cálculo del Índice de Masa Corporal (IMC) permite identificar el nivel de obesidad de las personas, factor íntimamente relacionado con el riesgo de padecer enfermedades cardiovasculares, dislipidemias, diabetes, hipertensión, hipercolesterolemia (Kanhai, Kappelle, Van der Graaf, Uiterwaal y Visseren, 2012). Los valores óptimos, en los que el riesgo de patologías es muy bajo, oscilan entre 20 y $24,9 \mathrm{~kg} / \mathrm{m}^{2}$ (Colado, 2004). El incremento de la obesidad se relaciona de manera directa y proporcional con el síndrome metabólico, ambos son factores de riesgo de enfermedades como la diabetes tipo 2, la enfermedad arterial coronaria y cerebrovascular por arterioesclerosis (Ramírez y otros, 2011; Moreno, Monereo y Álvarez, 2000). La enfermedad cardiovascular es la principal causa de muerte e invalidez a nivel mundial tanto en países desarrollados como en países en vías de desarrollo (Michelotto y otros 2010; Villar, Maiques, Brotons, Torcal, Lorenzo, Vilaseca y otros, 2001). Los porcentajes de grasa óptimos oscilan entre 8 y $15 \%$ para hombre y entre 13 y $20 \%$ para mujeres (García, 2007).

En la Circunferencia de Cintura (CC) se refleja la grasa intraabdominal de las personas, factor asociado con aumentos significativos en los triglicéridos, las lipoproteínas de baja densidad (LDL), hiperlipidemia pospandrial, fibrinógeno, proteína C-reactiva, citosinas y disfunción endotelial y con niveles reducidos de lipoproteínas de alta densidad (HDL) y adiponectina, lo que se relaciona con un mayor riesgo de enfermedad cardiovascular, hiperglucemia, hipertensión arterial (HTA) y dislipidemia (Franch, 2008; Prado, Carmenate, Martínez, Díaz y Toledo, 2001). Se ha demostrado que altos niveles en ácidos grasos inhiben el proceso de la lipólisis, lo cual causa daño en tejidos y afecta los procesos fisiológicos del organismo (Aguilar, 2007; Power, Alfonso, Flicker, Hankey, Yeap y Almeida, 2011).

Valores por arriba de 88 en la CC para hombres y superiores a 102 para las mujeres incrementan el riesgo cardiometabólico caracterizado por dislipidemias e hiperglucemias, y aumentan la posibilidad de sufrir un evento coronario (Franch, 2008; Heyward, 2008). La evidencia indica que índices elevados de grasa abdominal generan recarga de las concentraciones de ácidos grasos, lo que provoca el incremento de la síntesis de lípidos, lipoproteínas y glucosa (Daza, 2002), esto conlleva la producción hepática de glucosa y la producción de lipoproteínas, promoviendo la resistencia hepática a la acción de la insulina, y el aumento de la síntesis de triglicéridos y lipoproteínas de baja densidad (Aguilar, 2007; Rodríguez, Sánchez y Martínez, 2002).

El IMC y la relación entre cintura y cadera (RCC) son las medidas antropométricas que mayor relación tienen con el perfil lipídico en hombres y mujeres, por lo que se considera que estos indicadores reflejan el nivel de riesgo de la enfermedad coronaria (Michelotto, Martins, Machado, Santos y Carvalho, 2010). Mientras la CC es un buen predictor de la aparición de Diabetes Mellitus, el IMC es un predictor importante de la aparición de 
enfermedad cardiovascular (Concepción, Arioza, Gonzales, Álvarez y Robaina, 2007). Se afirma que la obesidad es la causa principal de una gran variedad de alteraciones metabólicas entre las que se incluyen las dislipidemias, especialmente la hipertrigliceridemia y HDL colesterol bajo, HTA y diabetes mellitus tipo 2 (Arruda y otros, 2010; Trejo, 2004). Es importante señalar que la hipertensión arterial (HTA) es seis veces más frecuente en obesos que en individuos con peso normal (Sirvent y Garrido, 2009). También la HTA se asocia con factores metabólicos como la hipercolesterolemia, la hipertrigliceridemia, el colesterol HDL bajo, la obesidad y la diabetes (Concepción y otros 2007; Daza, 2002).

El impacto de la obesidad sobre la tensión arterial y los niveles de lípidos está determinado por la localización de la grasa corporal. La distribución central de la grasa se asocia de manera significativa con la resistencia a la insulina, en comparación con la adiposidad general, ya que los depósitos centrales de grasa son metabólicamente más activos que los periféricos y más sensibles a la lipólisis inducida por catecolaminas, pero menos a la acción antilipolítica de la insulina (Concepción, Arioza, Gonzales, Álvarez y Robaina, 2007; Wells y Victoria, 2005).

El registro de indicadores relativos al IMC, porcentaje de grasa y RCC es fundamental para evaluar la salud de las personas y para predecir la aparición de patologías de tipo cardiometabólicas. Sobre la base de la evidencia expuesta, el propósito de este estudio fue analizar las evaluaciones antropométricas realizadas a los usuarios (as) del Centro de Acondicionamiento Físico (CAF) de la Escuela de Ciencias del Movimiento Humano y Calidad de Vida (CIEMHCAVI) durante los dos primeros años de funcionamiento de este centro, para determinar el perfil antropométrico de los mismos y su relación con la salud.

\section{Metodología}

Se trató de un estudio de tipo descriptivo-correlativo en el que se analizó el comportamiento de variables antropométricas relacionadas con la salud.

\section{Unidades de observación.}

Se analizaron 563 expedientes correspondientes a los usuarios del CAF de la CIEMHCAVI durante los dos primeros años de este centro, lo que equivale a un $56,3 \%$ de la población. La información analizada correspondió a 315 hombres, cuya edad promedio fue de $28.58 \pm 11.39$ años, y 248 mujeres, con una edad promedio de $30.35 \pm 11.39$ años. El formato de protocolo de cada expediente incluía información general sobre los usuarios (as) del CAF, así como información relativa a la idoneidad de los mismos para realizar actividad física.

\section{Procedimiento.}

Se revisaron 1000 expedientes con la información correspondiente a la medición de las variables antropométricas de los (as) usuarios (as) del CAF de la CIEMHCAVI. Esto correspondió a la población total. Todas las mediciones y programas de ejercicio fueron realizados por promotores de la salud graduados a nivel universitario. Posteriormente, se seleccionaron los expedientes que contenían la información completa sobre las variables antropométricas requeridas para los efectos del estudio y con la valoración positiva de idoneidad para el ejercicio físico mediante el Cuestionario de Aptitud para la Actividad Física-Par-Q (Canadian Society for Exercise Physiology, 2002). Como paso final, se procedió con la construcción de la base de datos y se generó los análisis estadísticos congruentes con el propósito del estudio. 


\section{Análisis estadístico.}

Se calculó valores promedio y desviaciones típicas. Asimismo, se realizó análisis de $t$ student para grupos independientes en los casos en que se contrastó las variables antropométricas según el sexo de los participantes. Se aplicó análisis de varianza univariados para estudiar el comportamiento de las variables en función de la edad. Los cálculos post hoc se basaron en el test Scheffee. Finalmente, se determinó la relación entre las variables estudiadas por medio del procedimiento de correlaciones bivariadas de Pearson y se realizó análisis de $\mathrm{Chi}^{2}$ cuando se trató del contraste de proporciones. Para todos estos análisis se utilizó el paquete estadístico PASW 17.0 para Windows. El valor crítico establecido para las decisiones estadísticas correspondió a una $p \leq 0.05$.

\section{Resultados}

Más del $50 \%$ de hombres y mujeres se ubican en la categoría de peso normal (ver tabla 1). El contraste entre la proporción de hombres y mujeres en función de las categorías propuestas no evidenció diferencias significativas $\left(\mathrm{Chi}^{2}=0,93, p>0,05\right)$. La distribución por sexo, en función del porcentaje de grasa, mostró que las mujeres comparadas con los hombres tienen porcentajes de grasa superiores $\left(\mathrm{Chi}^{2}=70,29, p \leq 0,05\right)$.

Tabla 1

Valores absolutos y relativos correspondientes a las categorías de índice de masa corporal y porcentaje de grasa según sexo

\begin{tabular}{lcccc}
\multicolumn{1}{c}{ Categorías } & Femenino & \% de grasa & Masculino & $\%$ de grasa \\
\hline Bajo peso $<18,5$ & $6(3,3 \%)$ & 19,92 & $7(3,1 \%)$ & 1,14 \\
Normal $18,5-24,9$ & $96(53,2 \%)$ & 23,84 & $127(56,7 \%)$ & 11,96 \\
Sobrepeso $25,0-29,9$ & $69(32,6 \%)$ & 30,51 & $76(33,9 \%)$ & 20,23 \\
Obesidad (1) $30,0-34,9$ & $15(8,2 \%)$ & 34,74 & $11(4,9 \%)$ & 27,95 \\
Obesidad (2) $35,0-39,9)$ & $6(3,3 \%)$ & 36,51 & $2(0,9 \%)$ & 31,86 \\
Obesidad (3) $40 \geq$ & $1(0,5 \%)$ & 0 & $1(0,4 \%)$ & 0 \\
\hline
\end{tabular}

En función de la circunferencia de cintura, se encontró que el 87,5 y 94, $9 \%$ de las mujeres y hombres, respectivamente, presentaron niveles de riesgo entre bajo y muy bajo (ver tabla 2). El contraste de la distribución por sexo según el nivel de riesgo mostró que los hombres tienen un riesgo cardiovascular menor que las mujeres evaluadas ( $\mathrm{Chi}^{2}=16,88$, $p \leq 0,05)$.

Con excepción del IMC, en todas las variables medidas se encontró diferencias significativas entre hombres y mujeres. Las mujeres, comparadas con los hombres, son significativamente menos pesadas, pero evidencian valores promedio significativamente superiores en cuanto a porcentaje de grasa, circunferencia de cintura y de cadera (ver tabla 3 ). 
Tabla 2

Niveles de riesgo cardiovascular según sexo a partir de la circunferencia de cintura

\begin{tabular}{|c|c|c|c|}
\hline Niveles de riesgo & Femenino & Niveles de riesgo & Masculino \\
\hline Muy bajo $\leq 70$ & $76(30,5 \%)$ & Muy bajo $\leq 80$ & $139(44,3 \%)$ \\
\hline Bajo 70,1 - 89 & $142(57,0 \%)$ & Bajo 80,1 -99 & $159(50,6 \%)$ \\
\hline Alto $89,1-109$ & $28(11,2 \%)$ & Alto $99,1-120$ & $15(4,8 \%)$ \\
\hline Muy alto $109 \geq$ & $3(1,2 \%)$ & Muy alto $120 \geq$ & $1(0,3 \%)$ \\
\hline
\end{tabular}

De conformidad con la relación cintura cadera, el 97,8 \% de los hombres mostraron índices correspondientes a un bajo riesgo. En el caso de las mujeres, el $74,1 \%$ mostraron bajo riesgo cardiovascular (ver tabla 4).

Tabla 3

Valores promedio en general y comparación por sexo en las variables antropométricas evaluadas

\begin{tabular}{lccccc} 
Variables & General & Masculino & Femenino & $t$ & Sig. \\
\hline IMC & $24,7 \pm 4,21$ & $24,62 \pm 3,91$ & $24,94 \pm 4,57$ & $-0,76$ & 0,43 \\
Peso & $67,9 \pm 13,16$ & $72,14 \pm 12,87$ & $62,64 \pm 12,84$ & 8,44 & 0,00 \\
Grasa & $20,4 \pm 8,80$ & $15,39 \pm 7,24$ & $23,90 \pm 5,92$ & $-17,36$ & 0,00 \\
Cintura & $80,1 \pm 10,9$ & $82,66 \pm 10,29$ & $76,99 \pm 10,97$ & 6,31 & 0,00 \\
Cadera & $99,08 \pm 9,09$ & $98,01 \pm 8,30$ & $100,44 \pm 9,84$ & $-3,16$ & 0,00 \\
\hline
\end{tabular}

En cuanto a la relación cintura cadera, se registró diferencias significativas entre hombres y mujeres $(t=13,08 ; p<0.00)$. El valor promedio para los hombres fue de $0.84 \pm$ 0.07 y para mujeres de $0.76 \pm 0.06$. Las mujeres, comparadas con los hombres, mostraron un mayor riesgo de enfermedad cardiovascular (ver tabla 4).

Tabla 4

Niveles de riesgo según la relación cintura cadera por sexo

\begin{tabular}{lccc}
\multicolumn{1}{c}{ Nivel de riesgo } & Hombres & Nivel de riesgo & Mujeres \\
\hline Bajo riesgo $\leq 1$ & $308(97,8 \%)$ & Bajo riesgo $\leq 0,8$ & $177(74,1 \%)$ \\
Alto riesgo $>1,1$ & $7(2,2 \%)$ & Alto riesgo $>0,81$ & $62(25,9 \%)$ \\
\hline
\end{tabular}


En el caso de los hombres, la comparación de indicadores antropométricos según grupo de edad mostró que los hombres más jóvenes fueron quienes mostraron los valores promedio más bajos en todas las variables analizadas (ver tabla 5).

Tabla 5

Contraste entre los valores promedio de ingreso según grupos de edad en hombres

\begin{tabular}{|c|c|c|c|c|c|}
\hline Variable & $\leq 21$ años & 21,1 a 31años & 31,1 a 68años & $F$ & Sig. \\
\hline IMC & $23,50 \pm 3,79^{\mathrm{a}}$ & $24,75 \pm 3,24^{\mathrm{ab}}$ & $26,03 \pm 4,38^{\mathrm{b}}$ & 7,69 & 0,00 \\
\hline Peso & $68,11 \pm 11,27^{\mathrm{a}}$ & $74,01 \pm 12,57^{\mathrm{b}}$ & $74,62 \pm 13,71^{b}$ & 7,62 & 0,00 \\
\hline Grasa & $11,82 \pm 6,13^{\mathrm{a}}$ & $15,52 \pm 6,00^{b}$ & $19,41 \pm 7,50^{\mathrm{c}}$ & 23,83 & 0,00 \\
\hline Cintura & $79,00 \pm 9,48^{\mathrm{a}}$ & $82,73 \pm 8,36^{\mathrm{b}}$ & $86,86 \pm 11,67^{\mathrm{c}}$ & 15,16 & 0,00 \\
\hline Cadera & $95,73 \pm 7,47^{\mathrm{a}}$ & $99,65 \pm 9,13^{b}$ & $98,76 \pm 7,69^{\mathrm{b}}$ & 6,39 & 0,00 \\
\hline
\end{tabular}

En el grupo de mujeres estudiadas se encontró un perfil similar al de los hombres. En todos los indicadores antropométricos evaluados se registraron diferencias estadísticamente significativas en función de la edad. Las mujeres más jóvenes muestran los valores promedio más bajos.

Tabla 6

Contraste entre los valores promedio de ingreso según grupos de edad en mujeres

\begin{tabular}{lccccc} 
Variable & $\leq 21$ años & $21,1-31$ años & $31,1-68$ años & $F$ & Sig. \\
\hline IMC & $23,40 \pm 4,18^{\mathrm{a}}$ & $25,97 \pm 5,89^{\mathrm{b}}$ & $25,49 \pm 3,71^{\mathrm{ab}}$ & 4,38 & 0,01 \\
Peso & $59,07 \pm 10,19^{\mathrm{a}}$ & $65,11 \pm 15,32^{\mathrm{b}}$ & $63,74 \pm 10,84^{\mathrm{ab}}$ & 4,30 & 0,01 \\
Grasa & $25,07 \pm 5,96^{\mathrm{a}}$ & $27,57 \pm 6,15^{\mathrm{a}}$ & $27,74 \pm 5,58^{\mathrm{a}}$ & 3,35 & 0,03 \\
Cintura & $72,55 \pm 9,32^{\mathrm{a}}$ & $78,48 \pm 11,98^{\mathrm{b}}$ & $79,38 \pm 9,64^{\mathrm{b}}$ & 9,45 & 0,00 \\
Cadera & $97,46 \pm 7,44^{\mathrm{a}}$ & $102,75 \pm 11,49^{\mathrm{b}}$ & $101,41 \pm 8,78^{\mathrm{b}}$ & 5,91 & 0,00 \\
\hline \multicolumn{7}{l}{ Nota. Letras diferentes indican diferencias significativas $(p<05)$ entre los grupos. } & &
\end{tabular}

$\mathrm{El}$ análisis de correlaciones entre las variables estudiadas mostró relaciones positivas y significativas entre IMC y porcentaje de grasa $(r=0,56, p \leq 0,01)$, peso $(r=0,74, p<0,01)$, circunferencia de cintura $(r=0,84, p<0,01)$ y circunferencia de cadera $(r=0,79, p<0,01)$. Asimismo, el porcentaje de grasa correlacionó significativamente con peso $(r=0,15$, $p<0,01)$, circunferencia de cintura $(r=0,78, p<0,01)$ y circunferencia de cadera $(r=0,68$, $p<0,01)$. También, la circunferencia de cintura correlacionó con la circunferencia de cadera $(r=0,68, p<0,01)$. 


\section{Discusión}

En general, los resultados encontrados coinciden con lo reportado en la literatura científica relacionada con la temática analizada (Abernethy y otros, 2000). El valor promedio registrado para el IMC está en el rango considerado como saludable $\left(20 \mathrm{~kg} / \mathrm{m}^{2}-24,99 \mathrm{~kg} / \mathrm{m}^{2}\right)$ por el Colegio Americano de Medicina Deportiva en su Manual de Directrices para las Pruebas y Prescripción del Ejercicio (ACSM, 2009), lo cual refleja a una población sana desde el punto de vista fisiológico. No se registró diferencias entre hombres y mujeres, lo que es indicador de que se manejan cocientes similares entre peso y talla para ambos sexos. En relación con el peso, y como era de esperarse, los hombres mostraron ser significativamente más pesados que las mujeres, lo cual también tiene una explicación genética y fisiológica que se manifiesta en mayor cantidad de masa magra y mayor peso óseo (Berdasco, 2002).

El promedio general en cuanto a porcentaje de grasa también se ubicó en rangos definidos como normales (Bellido y De Luis, 2006), situación que también refleja a una población sana de acuerdo a los parámetros definidos por la American Heart Asociation (AHA, 2013). Sin embargo, las mujeres, en comparación con los hombres, mostraron porcentajes de grasa significativamente superiores, lo cual es explicable por cuanto, desde el punto de vista bioquímico y fisiológico, la acumulación de grasa es mayor en el sexo femenino (Heymsfield y otros, 2005). También los rangos en que se encuentran estos valores tanto para hombres como para mujeres son coincidentes con lo que se reporta en la literatura científica (Michelotto y otros, 2010). La dimensión de la cintura es menor en las mujeres que en los hombres, aunque el diámetro de la cadera es menor en los hombres. Estos datos también son consistentes con lo reportado en la literatura (Aguilar, 2007). Este fenómeno se relaciona con la mayor disposición de la mujer para acumular grasa (Alvero y otros, 2011) y tiene que ver con la necesidad que tiene la mujer de responder a su condición de fémina en el caso del parto (Bahamondes y otros 2012). Esta situación es influida por las condiciones genéticas y por las condiciones sociales que condicionan una vida sedentaria en las mujeres, lo cual facilita la acumulación de grasa (Casanueva y otros 2008).

En el caso de la edad, los resultados encontrados indican que esta variable es un factor que se asocia negativamente en los indicadores antropométricos IMC, grasa corporal, CC y diámetro de cadera, lo cual también es congruente con lo que se reporta en la literatura científica (Gómez y otros, 2012). Los datos recolectados indican que con el paso de los años, y con el proceso de envejecimiento implicado, tiende a aumentar el IMC, la acumulación de grasa y consecuentemente la CC y el diámetro de la cadera (Concepción y otros, 2007). Esto significa que, conforme se vayan ganando años, es preciso tener en cuenta que, asociado a ello, la respuesta bioquímica y fisiológica del organismo favorece la acumulación de grasa, con los consecuentes problemas asociados (Wilmore y Costill, 2007). Esta realidad permite subrayar el papel que juega la evaluación antropométrica como recurso para diagnosticar el comportamiento de indicadores que podrían atentar contra la salud de las personas.

Los problemas asociados con la edad se acentúan de manera particular a partir de los 30 años de edad, lo cual también es congruente con lo reportado en la literatura (López y Virgili, 2012). Entre los 30 y 50 años el organismo empieza a perder de forma degenerativa la masa muscular y fuerza, lo que favorece la acumulación de grasa con las consecuencias negativas para la salud (Gómez y otros, 2012). Esta tendencia se observa tanto en el caso de los hombres como en el de las mujeres. 
A pesar de que el mayor porcentaje de las personas evaluadas tienen normopeso, también un porcentaje importante mostró sobrepeso (Abernethy y otros, 2000), situación que alerta la atención, considerando las consecuencias que tiene para la salud la condición de sobrepeso, entre las que se pueden mencionar la diabetes, hipertensión, entre otros problemas cardiovasculares (Arpa y González, 2009). La valoración del riesgo cardiovascular a partir de la CC mostró porcentajes congruentes con lo registrado en la literatura científica (Aguilar, 2007). La mayoría de las personas evaluadas mostraron niveles de riesgo entre bajo y muy bajo, lo cual habla del buen estado de salud de las personas evaluadas. Desde el punto de vista de las posibilidades cardiovasculares, un buen estado de salud significa capacidad de trabajo, funcionalidad social y energía (Arruda y otros 2010), todas ellas condiciones necesarias para una buena calidad de vida y bienestar (Dishman y otros 2004). Las mujeres, comparadas con los hombres, mostraron un mayor nivel de riesgo cardiovascular a partir de la CC, lo que también es consistente con lo reportado en la literatura científica (Berdasco, 2002).

Los valores promedio relativos al porcentaje de grasa fueron mayores en las mujeres que en los hombres, hecho también consistente con lo que se reporta en la investigación científica (Alvero y otros, 2011). La RCC, a pesar de corresponder a un cociente normal tanto para hombres como para mujeres, evidenció conforme lo indicado en la literatura (Aguilar, 2007) que los hombres tienen un mayor cociente en la relación cintura cadera. Contrario a lo que se reporta en la investigación, las mujeres mostraron un mayor riesgo cardiovascular que los hombres a partir del contraste de la relación cintura cadera (Sabán, 2012). El cociente entre estas dos variables antropométricas refleja la acumulación de grasa (Daza, 2002), con las consabidas consecuencias que esto tiene para la salud, especialmente con las patologías de tipo cardiovascular (Power y otros, 2011).

En el análisis de la dinámica que caracteriza la asociación entre indicadores antropométricos IMC, porcentaje de grasa y RCC, se encontró asociaciones positivas y significativas, lo que es consistente con el comportamiento fisiológico del organismo (Arpa y González, 2009). Como era de esperarse, conforme aumenta el IMC, aumenta también el porcentaje de grasa, la CC, el diámetro de la cadera y el cociente resultante de la RCC.

En general, los resultados encontrados en este estudio muestran a un grupo de personas en su mayoría sanas desde el punto de vista de los indicadores antropométricos evaluados. Se ha confirmado una vez más el rol que juega la edad en los procesos asociados a los aumentos en el IMC, porcentaje de grasa y relación cintura cadera. Esta situación podría servir de alerta para las personas y considerar que, conforme los años van pasando, se hace cada vez más necesario invertir tiempo en programas de ejercicio físico sistemáticos y controlados, para promover un mejor estado de salud o mantener el existente. En este sentido, es importante recordar que el ejercicio físico, amén de los beneficios fisiológicos que tiene sobre el organismo, también se vincula con beneficios psicosociales (Stein, Molinero, Salguero, Corrêa y Márquez, 2008). Entre ellos se pueden mencionar el aumento de la autoestima y confianza en sí mismo, la disminución de los niveles de ansiedad, ira, angustia, depresión, el incremento de la independencia en personas mayores, también reduce la fatiga y se percibe una sensación de bienestar y placer, confirmado por la fisiología, al liberar endorfinas en el torrente sanguíneo (Serrato, 2008). 


\section{Referencias Bibliográficas}

Abernethy, P., Olds, T., Eden, B., Neill, M. y Baines, L. (2000). Antropometría, salud y composición corporal. Armenia, Colombia: Editorial Kinesis.

Aguilar, C. (2007). Adiposidad abdominal como factor de riesgo de enfermedades crónicas. Salud pública de México, 49, 313-316. Recuperado de http://www.redalyc.org/articulo.oa?id=10649124

Alvero, J., Giner, L., Alacid, F., Rosety, M. y Ordóñez, F. (2011). Somatotipo, masa grasa y muscular del escalador deportivo Español de elite. International Journal of Morphology, 29(4), 1223-1230. http://dx.doi.org/10.4067/S0717-95022011000400026

American College of Sports Medicine. (2009). ACSM's Guidelines for Exercise Testing and Prescription. ( $8^{\circ}$ ed.). Philadelphia, USA: Editorial Lippincot Williams \& Wilkins.

American Heart Association. (2013). 2013 AHA/ACC Guideline on Lifestyle Management to Reduce Cardiovascular Risk: A Report of the American College of Cardiology/American Heart Association Task Force on Practice Guidelines. Texas, USA: American Heart Association, Inc.

Arpa, A. y González, O. (2009). Diferentes formas de valorar el sobrepeso o la obesidad y su relación con el síndrome metabólico. Revista Cubana de Medicina Militar, 38(2). Recuperado de http://scielo.sld.cu/scielo.php?pid=S0138$\underline{65572009000200003 \& \text { script }=\text { sci_arttext }}$

Arruda, M., Martins, R., Machado, E., Santos de Moraes, E. y De Carvalho, T. (2010). Relación de indicadores antropométricos con factores de riesgo para enfermedad cardiovascular. Arquivos Brasileiros de Cardiologia, 94(4), 462-469. Recuperado de http://www.scielo.br/pdf/abc/v94n4/es_aop00610.pdf

Bahamondes, C., Cifuentes, B., Lara, E. y Berral, F. (2012). Composición corporal y somatotipo en fútbol femenino. Campeonato sudamericano sub-17. International Journal of Morphology, 30(2), 450-460. http://dx.doi.org/10.4067/S0717$\underline{95022012000200016}$

Bellido, D. y De Luis, D. (2006). Manual de nutrición y metabolismo. Madrid, España: Editorial Díaz de Santos S.A.

Berdasco, A. (2002). Evaluación del estado nutricional del adulto mediante la antropometría. Revista Cubana de Alimentación y Nutrición, 16(2). Recuperado de http://bvs.sld.cu/revistas/ali/vol16_2_02/ali09202.pdf

Canadian Society for Exercise Physiology. (2002). Physical Activity Readiness Questionnaire - PAR-Q. Recuperado de http://www.csep.ca/cmfiles/publications/parq/par-q.pdf

Casanueva, E., Kaufer, M., Perez, A. y Arroyo, P. (2008). Nutriología médica. (3ra ed.). Distrito Federal, México: Editorial Médica Panamericana. 
Colado, J. (2004). Fitness acuático. Barcelona, España: Editorial Paidotribo.

Concepción, L., Arioza, V., Gonzales, D., Álvarez, E. y Robaina, C. (2007). Correlación entre algunos indicadores del metabolismo lipídico y mediciones antropométricas en adultos con hipertensión arterial. Revista Cubana de Investigación Biomédica, 26(2). Recuperado de http://www.oalib.com/paper/1044160\#.U8bdSUBhv8Q

Daza, C. (2002). La obesidad: un desorden metabólico de alto riesgo para la salud. Revista Colombia Médica, 33(2). Recuperado de http://www.bioline.org.br/request?rc02014

Dishman, R., Washburn, R., \& Heat, G. (2004). Physical activity epidemiology. Illinois, USA: Human Kinetics

Franch, J. (2008). Obesidad intraabdominal y riesgo metabólico. Atención Primaria, 40(4). http://dx.doi.org/10.1157/13118063

García, A. (2007). Composición corporal. Ciencia y Deporte, 4(1). Recuperado de http://www.cienciaydeporte.net/index.php/numeros-anteriores/no-4/26articulos/60articulo.html

Gómez, A., Rodríguez, G., Vila-Maldonado, S., Casajus, A y Ara, I. (2012). Envejecimiento y composición corporal: la obesidad sarcopénica en España. Nutrición Hospitalaria, 27(1). Recuperado de http://www.nutricionhospitalaria.com/pdf/5502.pdf

Heyward. (2008). Evaluación de la aptitud física y prescripción del ejercicio. (5ºd.). Madrid, España: Editorial Médica Panamericana S.A.

Heymsfield, S., Lohman, T., Wang, Z., \& Going, S. (2005). Human body composition. Champaign, USA: Human Kinetics.

Kanhai, D., Kappelle, L., Van Der Graaf, Y., Uiterwaal, C., \& Visseren, F. (2012). The risk of general and abdominal adiposity in the occurrence of new vascular events and mortality in patients with various manifestations of vascular disease. International Journal of Obesity, 36(5), 695-702. http://dx.doi.org/10.1038/ijo.2011.115

López, R. y Virgili, M. (2012). Sarcopenia. Fresenius Kabi. Revista cuatrimestral No 5. Recuperado de http://www.freseniuskabi.es/nutricionenteral/pdf/infogeriatria/Infogeriatria_05.pdf\#pa $\mathrm{ge}=26$

Martínez, A., Carmenate, M., Díaz, M., Toledo, E., Prado, C., Padrón, R., Rodríguez, L., Wong, I., Moreno, R. y Moreno, V. (2009). Composición corporal y envejecimiento en la Habana, Cuba. Estudios de Antropología Biológica, IX. Recuperado de http://www.revistas.unam.mx/index.php/eab/article/view/30885

Michelotto, M., Martins, R., Machado, E., Santos, E. y Carvalho, T. (2010). Relación de indicadores antropométricos con factores de riesgo para enfermedad cardiovascular. Arquivos Brasileiros de Cardiologia, 94(4). Recuperado de http://www.scielo.br/pdf/abc/v94n4/es_aop00610. 
Moreno, B., Monereo, S. y Álvarez, J. (2000). La obesidad: epidemia del Siglo XXI. (2ºd.). Madrid, España: Editorial Díaz de Santos S.A.

Power, B., Alfonso, H., Flicker, L., Hankey, G., Yeap, B., \& Almeida, O. (2011). Body adiposity in later life and the incidence of dementia: The health in men study. Plosone, 6(3). http://dx.doi.org/10.1371/journal.pone.0017902.g001

Prado, C., Carmenate, M., Martínez, A., Díaz, M. y Toledo, E. (2001). Composición corporal e hipertensión arterial en ancianos de La Habana, Cuba. Revista digital Antropo. Recuperado de http://www.didac.ehu.es/antropo/0/0-2/prado.htm

Ramírez, M., Núñez, A., Velázquez, H., Tejeda, O., Cortés, B., Parra, A. y Rosas, V. (2011). Alteraciones cardiovasculares en una población infantil y su relación con trastornos metabólicos y antropométricos. Revista de Especialidades Médico- Quirúrgicas, 16(4), 199-207. Recuperado de http://www.medigraphic.com/pdfs/quirurgicas/rmq2011/rmq114c.pdf

Rodríguez, A., Sánchez, M. y Martínez, L. (2002). Síndrome metabólico. Revista Cubana de Endocrinología, 13(3). Recuperado de http://scielo.sld.cu/scielo.php?script=sci_arttext\&pid=S1561-29532002000300008

Sabán, J. (2012). Introducción al riesgo cardiovascular. Madrid, España: Editorial Díaz de Santos.

Serrato, M. (2008). Medicina del deporte. Bogotá, Colombia: Universal del Rosario.

Sirvent, J. y Garrido, R. (2009). Valoración antropométrica de composición corporal. Alicante, España: Editorial Universidad de Alicante.

Stein, A., Molinero, O., Salguero, A., Corrêa, M. y Márquez, S. (2008). Actividad física y salud percibida en pacientes con enfermedad coronaria. Cuadernos de Psicología del Deporte, 14. Recuperado de http://scielo.isciii.es/scielo.php?pid=S1578$\underline{84232014000100013 \& \text { script }=\text { sci_arttext }}$

Trejo, J. (2004). Epidemiología del síndrome metabólico y diabetes mellitus tipo 2: ¿El diluvio que viene?. Archivos de Cardiología de México, 74(2): 267-270. Recuperado de http://www.medigraphic.com/pdfs/archi/ac-2004/acs042ab.pdf

Villar, F., Maiques, A., Brotons, C., Torcal, J., Lorenzo, A., Vilaseca, J. y Banegas, J. (2001). Prevención cardiovascular en atención primaria. Atención Primaria, 28(2), 23-36. Recuperado de http://www.msssi.gob.es/ciudadanos/enfLesiones/enfNoTransmisibles/docs/cardiovasc ular.pdf

Wells, J., \& Victoria, C. (2005). Indices of whole-body and central adiposity for evaluating the metabolic load of obesity. International Journal of Obesity, 29(5), 483-489. Recuperado de http://www.readcube.com/articles/10.1038/sj.ijo.0802899 
Wilmore, J. y Costill, D. (2007). Fisiología del esfuerzo y el deporte. 6ta edición. Barcelona, España: Editorial Paidotribo.

World Health Association. (2000). Obesity: Preventing and managing the global epidemic. Geneva. Recuperado de http://apps.who.int/iris/bitstream/10665/42330/1/WHO_TRS_894.pdf?ua=1

Recepción: 05 de junio del 2014.

Corrección: 22 de julio del 2014.

Aceptación: 29 de julio del 2014.

Publicación: 31 de agosto del 2014. 